\title{
An unusual mycobacterial infection
}

\author{
David Sotello MD, Paula McKenzie MD, Marcella Rivas MD, Richard Winn MD
}

\begin{abstract}
The frequency of atypical mycobacterial or nontuberculous mycobacterial (NTM) infections has increased during the last three decades with the emergence of HIVIAIDS and more use of immunosuppressive treatments. We present a case of pulmonary mycobacterial infection secondary to Mycobacterium kansasii in a patient with chronic obstructive pulmonary disease (COPD) and malnutrition. M. kansasii is a ubiquitous organism, most commonly found in the southern and central regions of the US. It can occur as a colonizer, but when it produces disease it usually involves the lung. The American Thoracic Society (ATS) and Infectious Diseases Society of America (IDSA) have issued criteria to differentiate casual NTM isolation from true pulmonary NTM disease. Among the NTM infections, M. Kansasii is the pathogen which causes a clinical picture which most resembles pulmonary tuberculosis. It can produce a bronchiectasis, nodular lesions, and/or fibrocavitary infiltrates on x-rays. Treatment requires a rifampin based regimen, usually combined with isoniazid and ethambutol. If rifampin resistance is present, macrolides, quinolones, or sulfas are usually recommended.
\end{abstract}

Keywords: Mycobacterium, nontuberculous, atypical, diagnosis, treatment

\section{CASE}

A 56-year-old Caucasian man with past medical history of COPD secondary to tobacco abuse (40 packs per year of smoking) and chronic alcohol abuse presented to the Emergency Department with several months of increasing dyspnea, dry cough, fatigue, and malaise. He denied fever or other symptoms. He was using his wife's inhalers as needed without success. His blood pressure was 120/80 mm Hg; temperature $97.9^{\circ} \mathrm{F}$; heart rate 109 beats per minute, and respirations 21 per minute. He was 74" tall and weighed 55 $\mathrm{kg}$ (body mass index of $15.6 \mathrm{~kg} / \mathrm{m}^{2}$ ). On physical examination he looked in mild respiratory distress and

Corresponding author: David Sotello MD

Contact Information: david.sotello@ttuhsc.edu

DOI: 10.12746/swrccc 2014.0207.090 emaciated with temporal wasting. His chest examination showed symmetric chest wall movement with decreased expansion, decreased breath sounds, and diffuse wheezing and rhonchi. The rest of his physical examination was within normal limits. His blood counts and chemistry panel on admission were within normal limits. Admission arterial blood gas is shown in Table 1. Chest radiograph showed hyperinflation of the lungs with multiple bullae, especially in the upper lobes. No acute infiltrates were identified (Figure 1 ). The patient was initially treated for a COPD exacerbation with scheduled bronchodilators, systemic corticosteroids, and levofloxacin $750 \mathrm{mg}$ orally for 6 days without significant improvement. Purified protein derivative (PPD) testing was negative, QuantiFER$\mathrm{ON}$ test was indeterminate, and HIV screening was non-reactive. An acid-fast bacilli (AFB) stain (ZiehlNeelsen) showed a moderate number $(3+)$ of acidfast bacilli. The Infectious Diseases consult started the patient on rifampin, isoniazid, pyrazinamide, and 
ethambutol. Moxifloxacin and clarithromycin were added after reviewing the PPD and QuantiFERON results to include nontuberculous mycobacterial

Table 1. Admission arterial blood gas

Variable

Patient's value

$\begin{array}{lc}\mathrm{pH} & 7.43 \\ \mathrm{PCO} 2(\mathrm{~mm} \mathrm{Hg}) & 49.6 \\ \mathrm{PO} 2(\mathrm{~mm} \mathrm{Hg}) & 54.8 \\ \mathrm{HCO} 3(\mathrm{mmol} / \mathrm{L}) & 32.2 \\ \mathrm{PO} 2 / \mathrm{FiO} 2 \text { ratio } & 261 \\ \mathrm{~A}-\mathrm{a}(\mathrm{mm} \mathrm{Hg}) & 19.2 \\ \mathrm{FiO} 2(\%) & 21 \\ \text { O2 saturation }(\%) & 89 \\ \text { Lactate }(\mathrm{mmol} / \mathrm{L}) & 2.16\end{array}$

\section{Discussion}

Our report describes a case of atypical mycobacterial infection, also known as nontuberculous mycobacteria (NTM) infection, due to $M$. Kansasii. NTM comprise all mycobacterial species other than the obligate pathogens Mycobacterium tuberculosis and Mycobacterium leprae. Typically they are found in the environment in soil and treated water. More than 140 species have been described, but approximately 25 species cause NTM diseases. The most frequent risk factors for NTM infections are HIV/AIDS, immunosuppressive drugs, and preexistent lung diseases (e.g., COPD, cystic fibrosis). These bacteria can produce an extensive array of pathologies. Pulmonary involvement is the most common followed by lymphadenopathy, skin and other extrapulmonary sites, and disseminated disease. ${ }^{1}$ coverage. AFB culture reported almost three weeks later grew Mycobacterium kansasii.

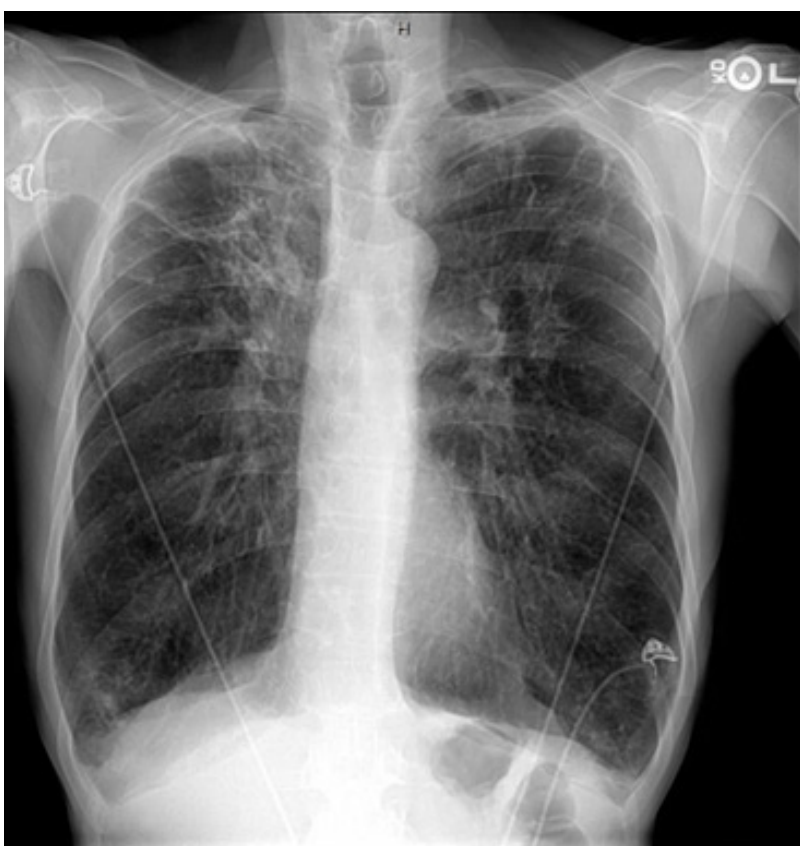

Figure 1. A PA chest radiograph shows hyperinflation of the lungs with multiple bullae, especially in the upper lobes; no acute infiltrates are seen.

The diagnosis of NTM can be challenging and cultures from non-sterile sources like the respiratory or digestive tract do not necessarily establish infection or disease. The ATS and IDSA have issued statements including a set of criteria to differentiate casual NTM isolation from true pulmonary NTM disease. These are summarized in Table 2. According to the previously mentioned criteria, the diagnosis is based on clinical, radiological, and microbiological evidence. Symptoms are nonspecific and include chronic cough with or without sputum production, hemoptysis, fatigue, and malaise. Less frequently patients have weight loss, fever, and night sweats which may indicate advanced disease. ${ }^{1-2}$ Radiological abnormalities follow two distinct patterns. The first is characterized by bronchiectasis and nodular lesions, mostly involv- 
ing the lingula and middle lobe; the second is characterized by fibrocavitary lesions that mostly involve the upper lobes and resemble pulmonary tuberculosis. Mixed patterns can occur. ${ }^{3}$

Microbiologic evidence requires at least three respiratory specimens. Sampling intervals should be at least several weeks apart, but the best interval between specimens has yet to be determined. At least two specimens should grow the same NTM species for a strong diagnosis. ${ }^{1}$ Smear microscopy is usually done in two steps. Samples are screened by fluorochrome staining, and positives are confirmed by Ziehl-Neelsen staining. ${ }^{1}$ Other molecular and bio- chemical techniques which might aid in the diagnosis of mycobacterial infections include radiometric cultures, detection of tuberculostearic acid (gas chromatography-mass spectrometry), mycobacterial antigens (enzyme-linked immunosorbent assays), DNA probes, and nucleic acid amplification systems such as PCR. ${ }^{4}$ Histological or cytological analysis can be useful in difficult cases, including patients who do not produce sputum. Bronchoalveolar lavage is probably more sensitive that sputum culture. NTM in extrapulmonary sites may require fine-needle aspirates or local excision to obtain microbiological evidence of the disease. 1,2 The choice of media for primary isolation largely determines the sensitivity. Liquid media

Table 2. Summary of the American Thoracic Society diagnostic criteria for pulmonary nontuberculous mycobacterial infection ${ }^{2}$

\section{Clinical}

1. Pulmonary symptoms, nodular or cavitary opacities on chest radiograph, or a high-resolution computed to mographic scan that shows multifocal bronchiectasis with multiple small nodules.

and

2. Appropriate exclusion of other diagnoses.

\section{Microbiologic}

1. Positive culture results from at least two separate expectorated sputum samples. If the results from the initial sputum samples are nondiagnostic, consider repeat sputum acid-fast bacillus (AFB) smears and cultures.

or

2. Positive culture results from at least one bronchial wash or lavage.

or

3. Transbronchial or other lung biopsy with mycobacterial histopathological features (granulomatous inflammation or AFB) and positive culture for NTM or biopsy showing mycobacterial histopathological features (granulomatous inflammation or AFB) and one or more sputum or bronchial washings that are culture positive for NTM.

4. Expert consultation should be obtained when NTM are recovered that are either infrequently encountered or that usually represent environmental contamination.

5. Patients who are suspected of having NTM lung disease but who do not meet the diagnostic criteria should be followed until the diagnosis is firmly established or excluded.

6. Making the diagnosis of NTM lung disease does not, per se, necessitate the institution of therapy, which is a decision based on potential risks and benefits of therapy for individual patients. 
are, in general, more sensitive than solid media, such as Lowenstein-Jensen, Ogawa, Coletsos, and Middlebrook $7 \mathrm{H} 10 / 7 \mathrm{H} 11 .{ }^{1}$ NTM have different levels of virulence depending on the species. Patients with preexisting lung cavity or bronchiectasis can be colonized with NTM and have persistently positive NTM cultures. This is why current ATS microbiologic criteria may be useful for virulent NTM, but more demanding criteria might be preferable for less virulent NTM. ${ }^{5}$ $M$. kansasii is the second most NTM responsible for human disease (Table 3). It is a slow growing, photochromogenic mycobacterium usually recovered from tap water, occasionally from river or lake water, and rarely from soil and animals. Phylogenetic and molecular analyses have identified seven different subtypes. Subtype 1 is the most frequently type isolated from humans, followed by subtype 2 . The clinical picture in pulmonary disease is similar to M. tuberculosis. ${ }^{6}$ Infection probably occurs by aerosol. ${ }^{7}$ Identification of isolates is usually made with high sensitivity and specificity DNA probes. ${ }^{7}$

In the United States M. kansasii infections occur more frequently in the southern and central regions in a pattern described as an inverted "T" (Texas, Louisiana, Florida, Illinois, Kansas and Nebraska). M. kansasii usually affects middle-age white men but can

Table 3. Non-tuberculous mycobacteria (NTM) that cause pulmonary infections in approximate order of prevalence $^{3}$
M. avium-intracellulare
M. kansasii
M. xenopi
M. fortuitum
M. chelonae
M. malmoense
M. gordonae
M. szulgai
M. simae
M. scrofulaceum
M. genavense

affect adults of any age, race or sex. Specific risk factors for M. kansasii infection include pneumoconiosis, COPD, previous mycobacterial disease, malignancy, and alcoholism. The combination of HIV and silicosis increases the susceptibility to infection. In HIV patients with high CD4 counts $M$. kansasii is usually associated with preexisting cavities; in patients with low CD4 counts it is often associated with disseminated disease. Initiation of empiric treatment may be necessary in patients with HIVIAIDS due to the potential for a rapidly fatal outcome. In patients with untreated disease, extensive lung destruction can occur.

There have been no randomized trials evaluating treatment regimens. ${ }^{2}$ Due to clinical similarities, patients with $M$. kansasii infection are frequently started on treatment for M. tuberculosis which overlaps with the current recommendations for treatment of $M$. kansasii lung disease, which include isoniazid (300 mg/day), rifampin (600 mg/day), and ethambutol $(15 \mathrm{mg} / \mathrm{kg} /$ day $)$ given daily for 18 months with at least 12 months of negative sputum cultures. M. kansasii is usually resistant to pyrazinamide. Successful therapy is considered by some experts to require documentation of negative sputum cultures for 12 months. Rifampin is the most important component in the treatment for $M$. kansasii. These isolates are less susceptible in vitro to isoniazid and streptomycin but are still susceptible to the achievable blood levels of these drugs. For this reason laboratory reports with resistance to low concentrations of these medications have no therapeutic significance as long as a rifampin containing regimen is used. In patients who are intolerant to one of the above mentioned drugs, clarithromycin can be used as a substitute. Shorter treatment courses have shown to be effective, but some of them show higher relapse rates. In patients infected with rifampin-resistant $M$. kansasii, a threedrug regimen is recommended based on in vitro susceptibilities, including clarithromycin or azithromycin, moxifloxacin, ethambutol, sulfamethoxazole, or streptomycin. Close clinical monitoring with frequent sputum culture should be done throughout therapy. The treatment regimen for disseminated disease is the same as for pulmonary disease.$^{2,7,8}$ 
In summary, NTM infection is a difficult diagnosis, and other infections have to be considered. This usually delays the diagnosis and the initiation of treatment. In the presented case the most likely predisposing factors for $\mathrm{M}$. kansasii infections were severe COPD with evidence of multiple preexistent bullae and malnutrition (BMI 15.6). Physicians should have high suspicion for NTM infections in patients with known predisposing conditions and expert consultation should be requested after obtaining a positive culture for treatment recommendations. More studies evaluating faster identification methods and shorter treatment regimens are needed in the field of NTM infections.

Author Affiliations: David Sotello and Marcella Rivas are residents in internal medicine at TTUHSC. Paula Mckenzie is a fellow in infectious disease at TTUHSC. Richard Winn is an infectious disease specialist in the department of internal medicine at TTUHSC.

Submitted: $5 / 6 / 2014$

Accepted: 6/2/2014

Reviewers: Steven Berk MD

Published electronically: 7/13/2014

Conflict of Interest Disclosures: None
5. Alvarez-Uria G. Lung disease caused by nontuberculous mycobacteria. Curr Opin Pulm Med 2010 May;16(3):251-6

6. Teillard C, et al. Clinical implications of Mycobacterium kansasii species heterogeneity: Swiss National Survey. J Clin Microbiol 2003 Mar; 41(3):1240-4.

7. Griffith DE. Management of disease due to Mycobacterium kansasii. Clin Chest Med 2002 Sep; 23(3):613-21.

8. Esteban J, Ortiz-Pérez A. Current treatment of atypical mycobacteriosis. Expert Opin Pharmacother 2009 Dec; 10(17):278799.

\section{REFERENCES}

1. Van Ingen J. Diagnosis of nontuberculous mycobacterial infections. Semin Respir Crit Care Med 2013 Feb; 34(1):103-9. 2. Griffith DE, Aksamit T, Brown-Elliott BA, et al; ATS Mycobacterial Diseases Subcommittee; American Thoracic Society; Infectious Disease Society of America. An official ATS/IDSA statement: diagnosis, treatment, and prevention of nontuberculous mycobacterial diseases. Am J Respir Crit Care Med 2007; 175(4):367-416.

3. Ellis SM. The spectrum of tuberculosis and non-tuberculous mycobacterial infection. Eur Radiol 2004 Mar; 14 Suppl 3:E3442.

4. Wright PW, Wallace RJ Jr, Wright NW, Brown BA, Griffith DE. Sensitivity of fluorochrome microscopy for detection of Mycobacterium tuberculosis versus nontuberculous mycobacteria. $\mathrm{J}$ Clin Microbiol 1998; 36(4):1046-1049. 